\title{
Circulating tumour cells as a biomarker for diagnosis and staging in pancreatic cancer
}

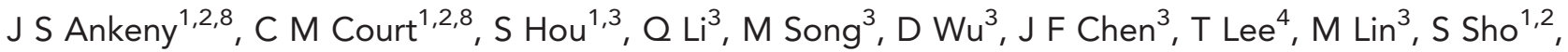 \\ M M Rochefort ${ }^{1}$, M D Girgis ${ }^{1}$, J Yao ${ }^{3}$, Z A Wainberg ${ }^{5,6}$, V R Muthusamy 5,7, R R Watson ${ }^{5,7}$, T R Donahue ${ }^{1,5}$, \\ O J Hines ${ }^{1,5}$, H A Reber ${ }^{1,5}$, T G Graeber ${ }^{3}$, H R Tseng ${ }^{3}$ and J S Tomlinson *,1,2,5 \\ ${ }^{1}$ Department of Surgery, University of California Los Angeles, 575 Westwood Plaza, Los Angeles, CA 90095, USA; ${ }^{2}$ Veteran's Health \\ Administration, Greater Los Angeles, Department of Surgery, 11301 Wilshire Boulevard, Los Angeles, CA 90073, USA; \\ ${ }^{3}$ Department of Molecular and Medical Pharmacology, Crump Institute for Molecular Imaging, California NanoSystems Institute, \\ University of California, Los Angeles, 570 Westwood Plaza, Los Angeles, CA 90095-1770, USA; ${ }^{4}$ California NanoSystems \\ Institute, University of California, 570 Westwood Plaza, Los Angeles, CA 90095, USA; ${ }^{5}$ UCLA Center for Pancreatic Diseases, \\ 575 Westwood Plaza, Los Angeles, CA 90095, USA; ${ }^{6}$ Department of Hematology/Oncology, University of California Los Angeles, \\ 575 Westwood Plaza, Los Angeles, CA 90095, USA and 'Department of Gastroenterology, University of California Los Angeles, 575 \\ Westwood Plaza, Los Angeles, CA 90095, USA
}

Background: Current diagnosis and staging of pancreatic ductal adenocarcinoma (PDAC) has important limitations and better biomarkers are needed to guide initial therapy. We investigated the performance of circulating tumour cells (CTCs) as an adjunctive biomarker at the time of disease presentation.

Methods: Venous blood (VB) was collected prospectively from 100 consecutive, pre-treatment patients with PDAC. Utilising the microfluidic NanoVelcro CTC chip, samples were evaluated for the presence and number of CTCs. KRAS mutation analysis was used to compare the CTCs with primary tumour tissue. CTC enumeration data was then evaluated as a diagnostic and staging biomarker in the setting of PDAC.

Results: We found $100 \%$ concordance for KRAS mutation subtype between primary tumour and CTCs in all five patients tested. Evaluation of CTCs as a diagnostic revealed the presence of CTCs in 54/72 patients with confirmed PDAC (sensitivity $=75.0 \%$, specificity $=96.4 \%$, area under the curve $(A \cup R O C)=0.867,95 \% \mathrm{Cl}=0.798-0.935$, and $P<0.001)$. Furthermore, a cut-off of $\geqslant 3$ CTCs in $4 \mathrm{ml} \mathrm{VB}$ was able to discriminate between local/regional and metastatic disease (AUROC $=0.885 ; 95 \% \mathrm{Cl}=0.800-0.969$; and $P<0.001)$.

Conclusion: CTCs appear to function well as a biomarker for diagnosis and staging in PDAC.

Pancreatic ductal adenocarcinoma (PDAC) is currently the fourth most common cause of cancer death in the United States, and is projected to be the second most common cause by 2030 (Rahib et al, 2014; Siegel et al, 2015). Its incidence approximates its mortality secondary to a 5 -year survival rate of $<6 \%$ (Siegel et al, 2015). These poor outcomes are undoubtedly related to a late presentation coupled with an aggressive biology (Yachida et al, 2010).
Initial diagnosis of PDAC typically utilises tissue acquisition via image-guided fine needle aspiration (FNA), most commonly by endoscopic ultrasound (EUS; Hawes et al, 2000). Unfortunately, these methods are expensive, inconvenient, require local expertise, and are not without risk to the patient (Cooper et al, 2013). In addition, for PDAC tumours, the abundance of stromal elements relative to cancer cells can lead to non-diagnostic biopsies. Thus, multiple needle passes and sometimes even repeated EUS/FNA is

\footnotetext{
*Correspondence: Dr JS Tomlinson; E-mail: jtomlinson@mednet.ucla.edu

${ }^{8}$ Authors share co-first authorship.
}

Received 5 January 2016; revised 18 February 2016; accepted 8 April 2016

(c) 2016 Cancer Research UK. All rights reserved 0007-0920/16 
necessary to obtain adequate tissue for diagnosis (LeBlanc et al, 2004). Clinical staging is based on cross-sectional imaging (CT or MRI) which does not have the necessary sensitivity to detect small-volume metastatic disease leading to routine under-staging. As evidence, $10-25 \%$ of patients who are initially believed to be resectable based on imaging are found to have metastases at surgical exploration (Diehl et al, 1998). Moreover, $\sim 80 \%$ of patients who undergo successful surgery will experience distant cancer recurrence, reflecting the presence of metastatic disease at the time of surgery (Cameron and He, 2015). Retrospectively, surgery may not be the appropriate initial therapy in these occult metastatic patients as it often delays administration of systemic therapy (Merkow et al, 2014). These data highlight the need for a biomarker that could improve the diagnosis and accuracy of staging at the time of disease presentation to better inform first-line therapy.

Circulating tumour cells (CTCs) are one promising biomarker that may be useful for these purposes in PDAC. Although CTCs have been studied in PDAC, they are not as well established as a biomarker in PDAC as compared with other solid cancers (Kurihara et al, 2008; Khoja et al, 2012). One reason may be the low sensitivity of current technology in detecting CTCs from peripheral blood in PDAC. Initial studies using the Food and Drug Administration (FDA) approved CellSearch Assay (Janssen Diagnostics, Raritan, NJ, USA) have confirmed the presence of CTCs in patients with PDAC, but in significantly lower overall numbers (Khoja et al, 2012) when compared with other epithelial cancers (Cristofanilli et al, 2004). In addition, it is unclear if CTCs identified across various studies are truly cells of tumour origin (Alix-Panabieres and Pantel, 2014). In PDAC, mutations in KRAS are among the most common found in any cancer and occur with an incidence of $>95 \%$ (Bryant et al, 2014). Moreover, $98 \%$ of the KRAS mutations found in PDAC are located in codon 12. Given the nearly uniform occurrence of KRAS mutations, tumour origin of captured CTCs can be inferred for most patients by sequencing a single gene. Unfortunately, the CellSearch Assay does not conveniently enable comprehensive molecular analysis of captured CTCs to confirm tumour origin or further inform the biology of metastasis.

Sensitivity has been improved with the development of new CTC detection and enumeration platforms (Alix-Panabieres and Pantel, 2014). The NanoVelcro platform utilises anti-EpCAMcoated nanosubstrates in conjunction with microfluidic chaotic mixers to improve CTC capture and identification (Wang et al, 2011; Chen et al, 2015). One advantage of the NanoVelcro platform is that it allows for the addition of tumour identification markers, such as CEA for PDAC (Girgis et al, 2011). In addition, NanoVelcro allows for seamless integration with laser capture micro-dissection (LCM) for single CTC isolation, referred to as NanoVelcro/LCM (Hou et al, 2013). The individually isolated CTCs can then be subjected to downstream molecular analyses, such as Sanger sequencing or next-generation sequencing.

In the present study, our goal was to evaluate CTCs as a biomarker for diagnosis and staging of PDAC at the time of disease presentation. To do this, we first developed a sensitive and specific method for PDAC CTC detection using the NanoVelcro platform in conjunction with high-resolution fluorescent microscopy and a multi-colour immunocytochemistry (ICC) approach. We validated our CTC definition using NanoVelcro/LCM by mutational analysis of KRAS codon 12, demonstrating concordance of KRAS mutation subtype between CTCs and primary tumour tissue. We then conducted CTC enumeration on 100 consecutive patients with suspicious pancreatic lesions or recent PDAC diagnosis and correlated these data with eventual diagnostic, pathologic, and staging information. Together, our results highlight the utility of CTCs as a liquid biopsy to better inform diagnosis and staging of PDAC, importantly, at the time of disease presentation.

\section{MATERIALS AND METHODS}

Calibration and optimisation of NanoVelcro Chips for PDAC. The performance of NanoVelcro Chips has previously been demonstrated and validated for several types of cancer, including prostate cancer and melanoma (Hou et al, 2013). However, we first sought to validate both the NanoVelcro and NanoVelcro/LCM chips, as well as our single-cell analysis, for pancreatic cancer using 4 PDAC cell lines obtained from American Type Culture Collection (ATCC, Manassas, VA, USA) with various cellular phenotypes (Supplementary Table S1). Details of cell culture and calibration are available in the Supplementary Methods. In brief, PDAC cells were spiked in healthy donor blood to create artificial blood samples, and run using the method outlined below and in the Supplementary Methods. For initial calibration, 200 cells from each cell line were spiked into 1-ml healthy donor blood and were processed on the NanoVelcro platform at various flow rates in triplicate to determine the optimum flow rate for capture efficiency (Supplementary Figure S1A). A flow rate of $1.0 \mathrm{ml} \mathrm{h}^{-1}$ was found to be the optimum flow rate, and was subsequently used for all patient samples (Supplementary Figure S1A). Using the same method, capture efficiencies for each of the 4 cell lines was calculated (Supplementary Figure S1B).

Patient recruitment and sample processing. Consecutive pretreatment patients with either suspicion for, or recently diagnosed, PDAC were approached regarding participation in our study between December 2012 and 2014, and enrolled in the study under University of California, Los Angeles IRB\#11-002112. Inclusion criteria were suspicious cystic or solid pancreatic lesions based on cross-sectional imaging or recent diagnosis of PDAC via biopsy, as well as willingness to give informed consent. Exclusion criteria included previous PDAC-directed treatment of any kind (surgical or non-surgical), active inflammatory bowel disease, synchronous malignancies, or other malignancy in the past 5 years. After a 5-ml waste to prevent epithelial cell contamination, $10 \mathrm{ml}$ venous blood (VB) was taken from a peripheral vein. Four millilitre was processed in parallel $2 \mathrm{ml}$ samples for enumeration studies and $6 \mathrm{ml}$ was cryopreserved. CA19-9 values were recorded when available. A database with demographic, pathologic, and relevant clinical outcome/survival variables was maintained in a prospective manner. All PDAC diagnoses were confirmed via biopsy or surgical pathology. Non-adenocarcinoma diseases were confirmed via biopsy or surgical pathology when possible. In some cases, intraductal papillary mucinous neoplasm was diagnosed clinically using a combination of cross-sectional imaging appearance, EUS characterisation, lack of malignant cells on FNA, and cyst fluid analysis.

Patient sample/NanoVelcro Chip preparation. In each $4 \mathrm{ml} \mathrm{VB}$ sample, red blood cells (RBCs) were lysed using a $0.15-\mathrm{M}$ Tris-ammonium chloride solution. Cold phosphate-buffered saline (PBS; Gibco, Carlsbad, CA, USA) was then added for termination of lysis and samples were subsequently centrifuged at $300 \mathrm{~g}$ for $10 \mathrm{~min}$ at $4{ }^{\circ} \mathrm{C}$. After removal of supernatant, cell pellets were re-suspended in cold RPMI-1640 (Cellgro, Manassas, VA, USA) supplemented with $5 \%$ cell-free foetal bovine serum (Gibco). Following a second centrifugation and removal of supernatant, cell pellets were re-suspended in PBS $+2 \%$ donkey serum (DS; Jackson Immunoresearch, West Grove, PA, USA). Biotinylated goat antiEpCAM (R\&D Systems, Minneapolis, MN, USA) was added to the samples and allowed to incubate at room temperature for $30 \mathrm{~min}$ with gentle agitation to prevent cell clumping. Following antibody incubation, samples were washed in PBS and re-suspended in 400- $\mu$ l PBS for chip loading. All experiments were carried out on parallel duplicate chips each running $200-\mu$ l of the re-suspended sample. NanoVelcro was assembled and operated as outlined in the 
Supplementary Materials and Methods and as previously described (Supplementary Figure S2; Hou et al, 2013). The prepared samples were injected into the device at a flow rate of $1.0 \mathrm{ml} \mathrm{h}^{-1}$, followed by $4 \%$ paraformaldehyde (PFA) at the same rate for fixation.

Immunocytochemistry (ICC) and chip mounting. Following disassembly of the device, processed chips were washed in PBS for $15 \mathrm{~min}$. Chips were blocked and cells permeabilised using PBS $+2 \%$ DS $+0.1 \%$ Triton X-100 (Sigma, St. Louis, MO, USA) for $15 \mathrm{~min}$. Chips were then incubated with a cocktail of primary antibodies containing two rabbit anti-pancytokeratin (CK) antibodies (Invitrogen, Carlsbad, CA, USA; Abcam, Cambridge, UK), one chicken anti-CEA antibody (Abcam), and two mouse antiCD45 antibodies (BD Pharmigen, Franklin Lakes, NJ, USA; Abcam) for $1 \mathrm{~h}$ at room temperature in PBS $+2 \% \mathrm{DS}$. Following primary antibody incubation, chips were washed again in PBS. Secondary antibody incubation was carried out in PBS $+2 \%$ DS for $1 \mathrm{~h}$ at room temperature using a cocktail of AlexaFluor-488 donkey anti-rabbit (Invitrogen), AlexaFluor-647 goat anti-chicken (Invitrogen), and AlexaFluor-555 donkey anti-mouse (Invitrogen). Chips were again washed in PBS and then attached to microscope slides with adhesive and mounted with cover slides using a DAPI mounting solution (Molecular Probes, Life Technologies, Carlsbad, CA, USA). Mounted slides were allowed to dry for $1 \mathrm{~h}$ before chip fluorescent scanning.

Chip scanning and CTC enumeration. Automated chip scanning with a Nikon Eclipse90i fluorescent microscope utilising NIS Elements 4.1 software (Nikon, Tokyo, Japan) was performed at $\times 40$ power to identify candidate cells. Higher $(\times 400)$ power manual imaging of candidate cells was then performed, and the results plotted on a fluorescence scatter plot to verify identity and count. When viewing, WBCs were defined as round/ovoid, $\mathrm{DAPI}+/ \mathrm{CD} 45+/ \mathrm{CK}-/ \mathrm{CEA}-$. CTCs were defined as round/ovoid, size $\geqslant 6-\mu \mathrm{m}, \mathrm{DAPI}+/ \mathrm{CD} 45-$, and $\mathrm{CK}+$ or CEA + (Figure 1B). Importantly, any $\mathrm{CD} 45$ positivity visible above background discounted a cell as being a CTC. Final CTC counts are represented as a total count per $4 \mathrm{ml} \mathrm{VB}$. CTC enumeration was carried out by the same, blinded researcher.

KRAS mutational analysis of single CTCs. Venous blood was collected from 5 PDAC patients in which CTC counts were high on enumeration studies and primary tumour tissue was available. These samples were used for single CTC KRAS mutational analysis. Samples were collected and processed as described above for RBC lysis, antibody incubation, assembly of NanoVelcro Chips, and ICC for identification (Supplementary Figure S3). However, 100\% ethanol replaced 4\% PFA for fixation and Hoechst-stain replaced DAPI for nuclear staining. Triton-X was excluded from antibody incubations to prevent any DNA damage. NanoVelcro/LCM was then used to allow for isolation of single CTCs and WBCs into individual Eppendorf tubes using an Arcturus-LMD device (Thermo Fisher Scientific, Waltham, MA, USA) attached to a Nikon Eclipse Ti fluorescent microscope (Supplementary Figure S4). Cell samples then underwent whole-genome amplification (WGA) using the REPLIg Single Cell Kit (Qiagen, Hilden, Germany), per the manufacturers protocol. WGA samples were then subjected to PCR amplification of a 300-base pair fragment surrounding KRAS codon-12 using Platinum PCR SuperMix High Fidelity kits (Invitrogen). The primers (Integrated DNA Technologies, Coralville, IA, USA) used were as follows: forward 5'-AAGGTACTGGTGGAGTATTTG- $3^{\prime}$ and reverse $5^{\prime}$-GTACT CATGAAAATGGTCAGAG- $3^{\prime}$. Each PCR reaction underwent 35 cycles with a denaturing step of $94{ }^{\circ} \mathrm{C}$ for $30 \mathrm{~s}$ followed by an annealing step at $55^{\circ} \mathrm{C}$ for $30 \mathrm{~s}$ and an elongation step at $68^{\circ} \mathrm{C}$ for $30 \mathrm{~s}$. The presence of KRAS PCR product was confirmed by gel electrophoresis on 2\%-agarose gels (Supplementary Figure S5). Samples were then sent for Sanger Sequencing by the UCLA
Genotyping and Sequencing Core on an Applied Biosystems 3730 DNA Analyser (Applied Biosystems, Foster City, CA, USA). The forward primer listed above for KRAS amplification was used as the sequencing primer in all reactions. Sequencing results were then analysed using FinchTV (Geospiza, Seattle, WA, USA).

Isolation of primary matched tumour tissue. Formalin fixed, paraffin embedded (FFPE) tumour blocks from the five patients with CTCs confirmed by Sanger sequencing were obtained. Ten serial sections of $5-\mu \mathrm{M}$ thickness were cut and placed on PEN-membrane slides (Leica, Wetzlar, Germany) followed by H\&E staining by the UCLA Translational Pathology Core Laboratory. Areas of tumour were identified and marked by a pathologist. These areas were then laser micro-dissected using the Palm Micro-Beam laser micro-dissection system (Carl Zeiss AG, Jena, Germany) to enrich the tumour content. DNA was then extracted and amplified using the REPLIg FFPE kit (Qiagen) according to the manufacturer's protocol. KRAS PCR and Sanger sequencing was then performed as previously described.

Statistical methods. All CTC numbers are reported as whole numbers in 4-ml of blood. Additional outlier analysis was performed with the Iterative Grubb's method $(\alpha=0.01)$. Differences in CTC number or CA19-9 level between non-adenocarcinoma and PDAC patients and those within the PDAC cohort were evaluated with a Mann-Whitney $U$-test given non-normal distribution. Comparisons across more than 2 groups were evaluated with a Kruskal-Wallis one-way ANOVA and the multiple comparisons test. Diagnostic performance of CTCs were evaluated using sensitivity, specificity, positive predictive value (PPV), negative predictive value (NPV), and positive likelihood ratio ( $+\mathrm{LR}$ ) calculations in addition to the use of receiver operating characteristic curves (ROCs) for determination of the area under the curve (AUROC) and overall discriminatory ability. A two-tailed $P$-value $<0.05$ was considered to be statistically significant. All statistical manipulations and calculations were performed with the assistance of GraphPad Prism6.0 (GraphPad Software, La Jolla, CA, USA).

\section{RESULTS}

Calibration and validation of NanoVelcro system and single-cell KRAS analysis. The NanoVelcro and NanoVelcro/LCM platforms were optimised and calibrated to detect PDAC CTCs utilising 4 PDAC cell lines (Supplementary Table S1). Calibration and capture efficiency data for each of the 4 PDAC cell lines are depicted (Supplementary Figure S1A and B). Immunocytochemistry definitions and KRAS mutational analysis were validated on artificial PDAC CTC samples (healthy donors' blood spiked with PDAC cell lines). AsPC-1, a homozygous PDAC cell line with a KRAS G12D mutation, and CFPAC-1, a heterozygous PDAC cell line with KRAS G12V mutation were utilised for KRAS mutational analysis. Batch cell DNA from each cell line was directly amplified for KRAS codon-12 and sequenced to verify published mutations and zygosity (Supplementary Figure S5). Twenty single cells from each cell line were then isolated via LCM. We were able to identify the G12D mutation in single AsPC-1 cells, and the G12V mutation in single CFPAC-1 cells (Supplementary Figure S5).

Study cohort. We successfully enrolled 108 patients as described in the Methods section (Figure 2). Eight patients were excluded (3-duodenal cancer, 3-concurrent second malignancy, 1-exacerbation of inflammatory bowel disease, and 1-refused informed consent). Thus, our study cohort consisted of 100 patients: 28 with non-adenocarcinoma diagnoses and 72 with a diagnosis of PDAC (Table 1). Of the 72 patients with PDAC, $4.2 \%$ had AJCC stage-I disease, $38.9 \%$ had stage-II disease, $19.4 \%$ had stage-III disease, and 
A

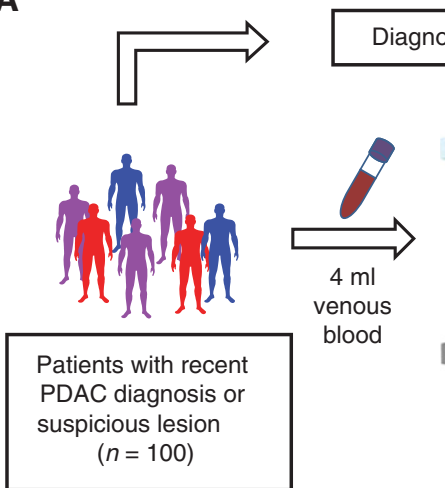

Diagnosis and staging information
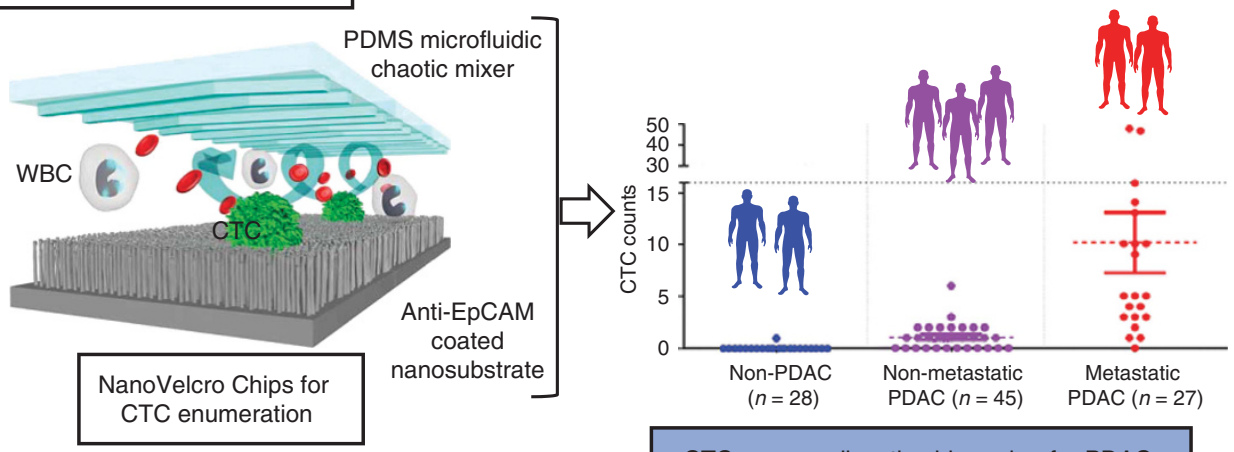

CTCs as an adjunctive biomarker for PDAC diagnosis and staging

B

1. 4-colour ICC staining

2. High-resolution fluorescent microscopy

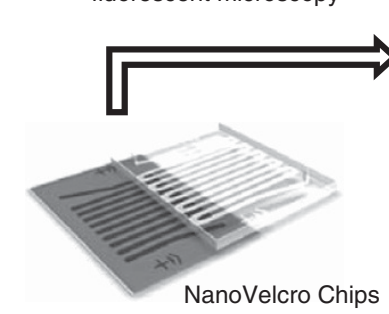

CTC markers and morphology enhance specificity
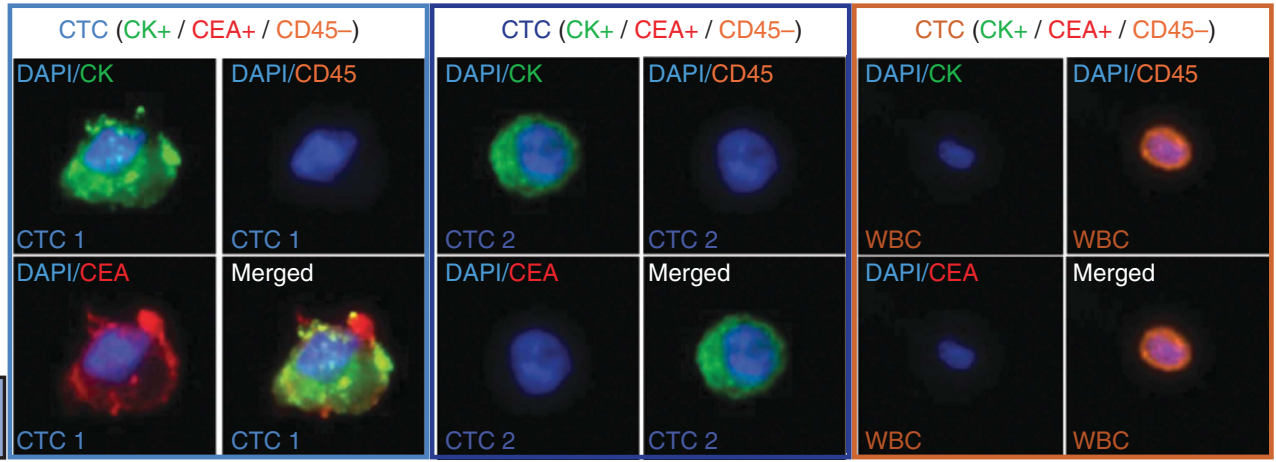

Figure 1. Study design and CTC detection principles. (A) Flow diagram depicting VB draws from our study cohort followed by CTC enumeration on NanoVelcro Chips and subsequent correlation with diagnostic and staging information. (B) Schematic depicting CTC identification via a 4-colour ICC approach in conjunction with high-resolution fluorescent microscopy. Representative images of 2 common CTC and WBC staining patterns are shown at $\times 400$ magnification.

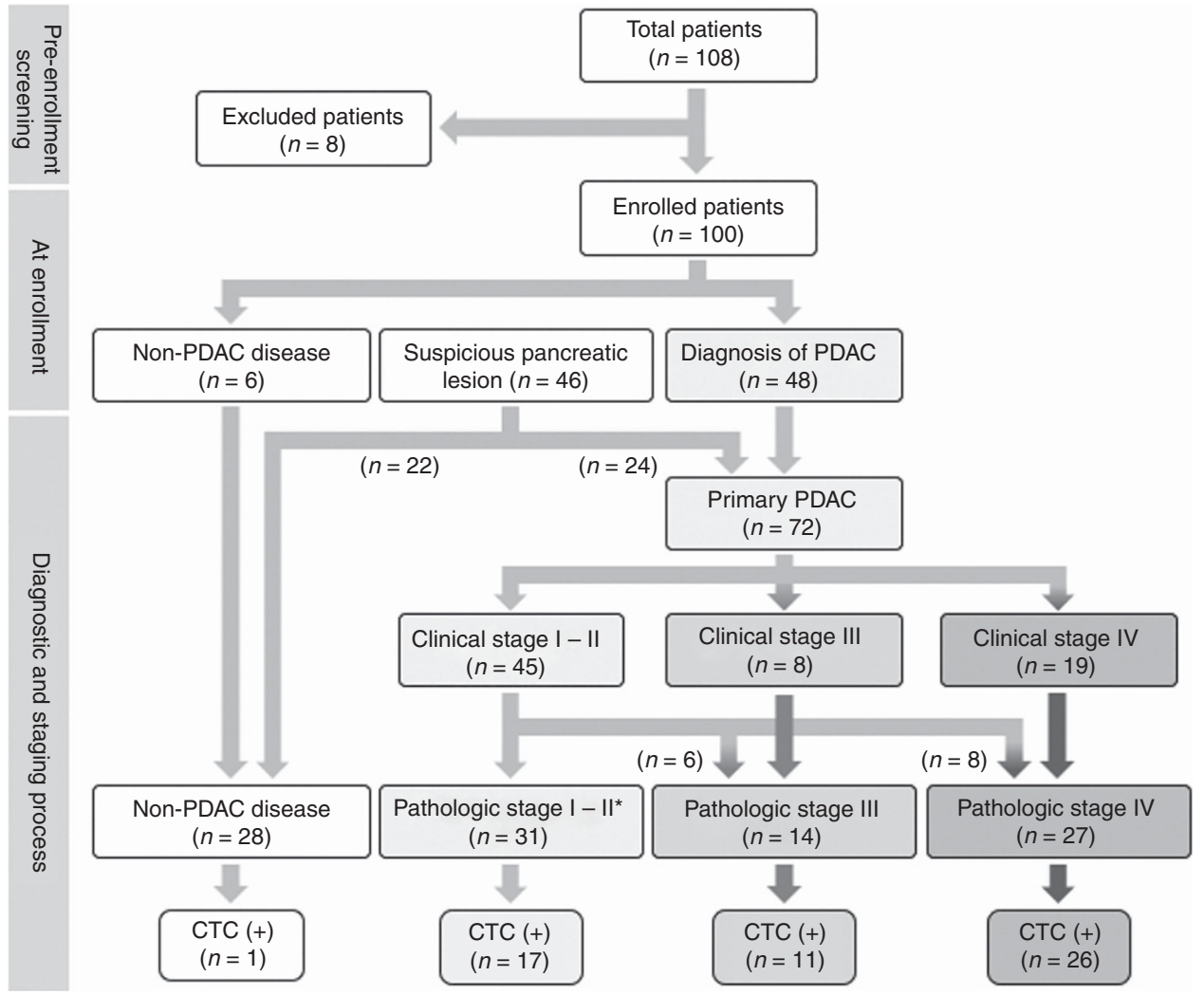

Figure 2. Study cohort characteristics. Diagnostic and staging flowchart of enrolled patients in the study. 
Table 1. Clinical and pathological characteristics associated with CTC counts

\begin{tabular}{|c|c|c|c|c|c|c|}
\hline Characteristic & Number (n) & О СТС & $\geqslant 1$ СТС & $\geqslant 2$ СТC & $\geqslant 3$ CTC & $\geqslant 5$ СТС \\
\hline Adenocarcinoma & 72 & $18(25.0 \%)$ & $54(75.0 \%)$ & $39(54.2 \%)$ & $29(40.3 \%)$ & $18(25.0 \%)$ \\
\hline $\begin{array}{l}\text { Stage } \\
\text { I } \\
\text { II } \\
\text { III } \\
\text { IV }\end{array}$ & $\begin{array}{c}3 \\
28 \\
14 \\
27\end{array}$ & $\begin{array}{c}3(100 \%) \\
11(39.3 \%) \\
3(21.4 \%) \\
1(3.7 \%)\end{array}$ & $\begin{array}{c}0 \\
17(60.7 \%) \\
11(78.6 \%) \\
26(96.3 \%)\end{array}$ & $\begin{array}{c}0 \\
9(32.1 \%) \\
6(42.9 \%) \\
24(88.9 \%)\end{array}$ & $\begin{array}{c}0 \\
2(7.1 \%) \\
4(28.6 \%) \\
23(85.2 \%)\end{array}$ & $\begin{array}{c}0 \\
1(3.6 \%) \\
2(14.3 \%) \\
15(55.6 \%)\end{array}$ \\
\hline $\begin{array}{l}\text { Node status } \\
- \\
+ \\
\text { NA }\end{array}$ & $\begin{array}{c}7 \\
20 \\
45\end{array}$ & $\begin{array}{c}5 \text { (71.4\%) } \\
7(35.0 \%) \\
-\end{array}$ & $\begin{array}{c}2(28.6 \%) \\
13(65.0 \%) \\
-\end{array}$ & $\begin{array}{c}2(28.6 \%) \\
6(30.0 \%) \\
-\end{array}$ & $\begin{array}{c}1(14.3 \%) \\
1(5.0 \%) \\
-\end{array}$ & $\begin{array}{c}1(14.3 \%) \\
0 \\
-\end{array}$ \\
\hline $\begin{array}{l}\text { Grade } \\
1 \\
2 \\
3 \\
\text { NA }\end{array}$ & $\begin{array}{c}6 \\
22 \\
11 \\
33\end{array}$ & $\begin{array}{c}2(33.3 \%) \\
7(31.8 \%) \\
3(27.3 \%) \\
-\end{array}$ & $\begin{array}{c}4(66.7 \%) \\
15(68.2 \%) \\
8(72.7 \%) \\
-\end{array}$ & $\begin{array}{c}3(50.0 \%) \\
9(40.9 \%) \\
6(54.5 \%) \\
-\end{array}$ & $\begin{array}{c}2(33.3 \%) \\
4(18.2 \%) \\
4(36.4 \%) \\
-\end{array}$ & $\begin{array}{c}2(33.3 \%) \\
3(13.6 \%) \\
1(9.1 \%) \\
-\end{array}$ \\
\hline $\begin{array}{l}\text { Surgery (PDAC) } \\
\text { Whipple } \\
\text { Distal panc } \\
\text { Ex lap stage III } \\
\text { Ex lap stage IV }\end{array}$ & $\begin{array}{c}22 \\
5 \\
6 \\
8\end{array}$ & $\begin{array}{c}9(40.9 \%) \\
3(60.0 \%) \\
0 \\
0\end{array}$ & $\begin{array}{l}13(59.1 \%) \\
2(40.0 \%) \\
6(100 \%) \\
8(100 \%)\end{array}$ & $\begin{array}{c}8(36.4 \%) \\
0 \\
4(66.7 \%) \\
7(87.5 \%)\end{array}$ & $\begin{array}{c}1(4.5 \%) \\
0 \\
4(66.7 \%) \\
7(87.5 \%)\end{array}$ & $\begin{array}{c}1(4.5 \%) \\
0 \\
2(33.3 \%) \\
4(50.0 \%)\end{array}$ \\
\hline Non-adenocarcinoma & 28 & 27 (96.4\%) & $1(3.6 \%)$ & 0 & 0 & 0 \\
\hline $\begin{array}{l}\text { Pathology/clinical Dx } \\
\text { IPMN (SB/MD) } \\
\text { MCN } \\
\text { Chronic panc } \\
\text { Serous cyst } \\
\text { Benign pancreas } \\
\text { PNET (G1) } \\
\text { Pseudocyst } \\
\text { Complex cyst }\end{array}$ & $\begin{array}{c}15(4 / 11) \\
2 \\
1 \\
4 \\
2 \\
2 \\
1 \\
1\end{array}$ & $\begin{array}{c}15(100 \%) \\
2(100 \%) \\
1(100 \%) \\
4(100 \%) \\
2(100 \%) \\
2(100 \%) \\
1(100 \%) \\
0\end{array}$ & $\begin{array}{c}0 \\
0 \\
0 \\
0 \\
0 \\
0 \\
0 \\
1(100 \%)\end{array}$ & $\begin{array}{l}0 \\
0 \\
0 \\
0 \\
0 \\
0 \\
0 \\
0\end{array}$ & $\begin{array}{l}0 \\
0 \\
0 \\
0 \\
0 \\
0 \\
0 \\
0\end{array}$ & $\begin{array}{l}0 \\
0 \\
0 \\
0 \\
0 \\
0 \\
0 \\
0\end{array}$ \\
\hline
\end{tabular}

37.5\% had stage-IV disease. Fourteen patients were initially in clinical stage I-II but were upstaged following surgical exploration secondary to finding locally advanced disease in 6 cases (stage-III) and metastatic (stage-IV) disease in the other eight cases (Table 1 and Figure 2). There were no statistically significant differences in patient sex or age between PDAC and non-adenocarcinoma diseases or in the differing PDAC stage groups.

KRAS mutational analysis of CTCs and tumour tissue. To molecularly validate our ICC criteria and provide evidence of pancreatic tumour origin of isolated CTCs, we performed KRAS mutational analysis on 5 patients that had both isolated CTCs and FFPE tumour tissue available (Figure 3A). Using our ICC criteria (round/ovoid, nucleus $+/ \mathrm{CK}+$, and/or CEA +/CD45-/size $\geqslant$ 6- $\mu \mathrm{m})$, which incorporates CEA in addition to $\mathrm{CK}$ as CTC identification markers (Figure 1B), both CTCs and WBCs were identified and isolated for mutational analysis. We isolated and sequenced a total of 44 CTCs and 21 WBCs from the 5 patients (Supplementary Table S2). We were able to identify codon-12 activating KRAS point mutations in $21 / 44$ (47.7\%) of the captured CTCs from the 5 patients, G12V (35G to T) in 1 patient and G12D ( $35 \mathrm{G}$ to $\mathrm{A}$ ) in 4 patients. We did not find heterogeneity in any patient with respect to KRAS subtype. In contrast, KRAS mutations were not identified in any of the 21 WBCs (Nucleus + /CK- and CEA-/CD45 + /any-size; Figure 3B). In addition, the KRAS mutation subtype (either G12V or G12D) of the FFPE tumour tissue matched that of the CTCs for all patients analysed.

CTCs in primary PDAC vs non-adenocarcinoma diseases. CTC enumeration was performed on VB from each of the patients enrolled in the study (Figure 1A and Supplementary Table S3). A series of images depicting our multi-marker staining for typical PDAC CTCs is depicted in (Figure 1B). In the PDAC group, CTCs were found to be present in 54/72 (75.0\%) patients (Figure $4 \mathrm{~A}$ ) ( median $=2$, range $=0-48$ ).

In the non-adenocarcinoma group, $1 / 28(3.6 \%)$ patients was found to have a single CTC in 4-ml VB (Figure 4A; median $=0$, range $=0-1$ ). This patient had a large, complex cyst on imaging and EUS, lack of malignant cells on FNA, and cyst fluid analysis with $\mathrm{CEA}<192 \mathrm{ng} \mathrm{ml}^{-1}$ and amylase of 274 units $^{-1}$. No confirmatory surgical pathology was available and no additional CTCs could be isolated from additional VB for KRAS mutational analysis.

CTCs as a diagnostic biomarker in PDAC. Calculation of Youden's J-statistic (J) at various CTC cut-off values established an optimal diagnostic performance for PDAC at 1 CTC in 4-ml VB (Supplementary Table S4). CTC presence thus demonstrated 75.0\%-sensitivity, 96.4\%-specificity, 98.2\%-PPV, 60.0\%-NPV, and a $+\mathrm{LR}$ of 21.00 for the diagnosis of primary PDAC. An ROC curve was then constructed and the AUROC was 0.867 (95\% $\mathrm{CI}=0.798-0.935$ and $P<0.001)$, thus illustrating the overall ability 


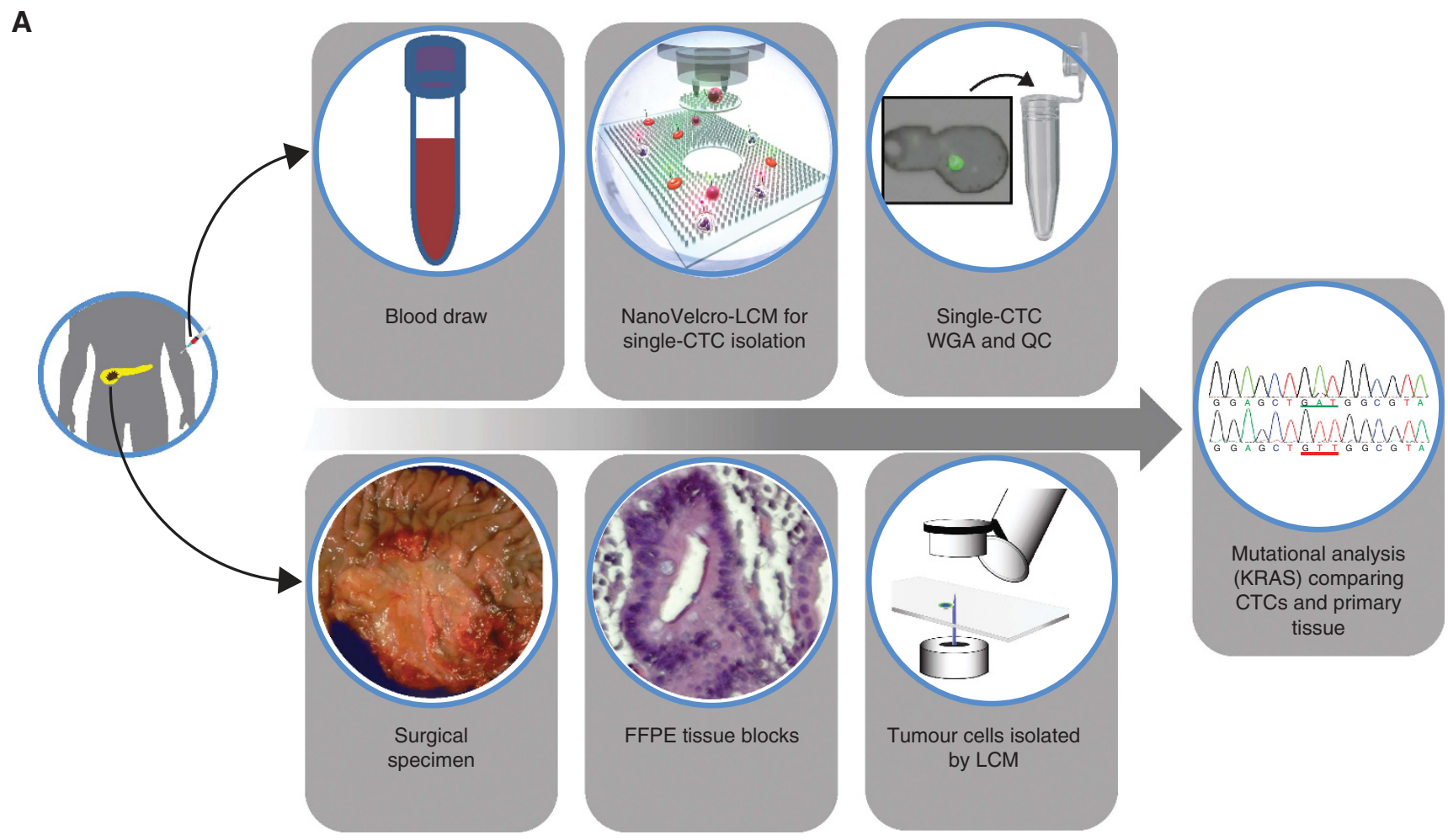

B
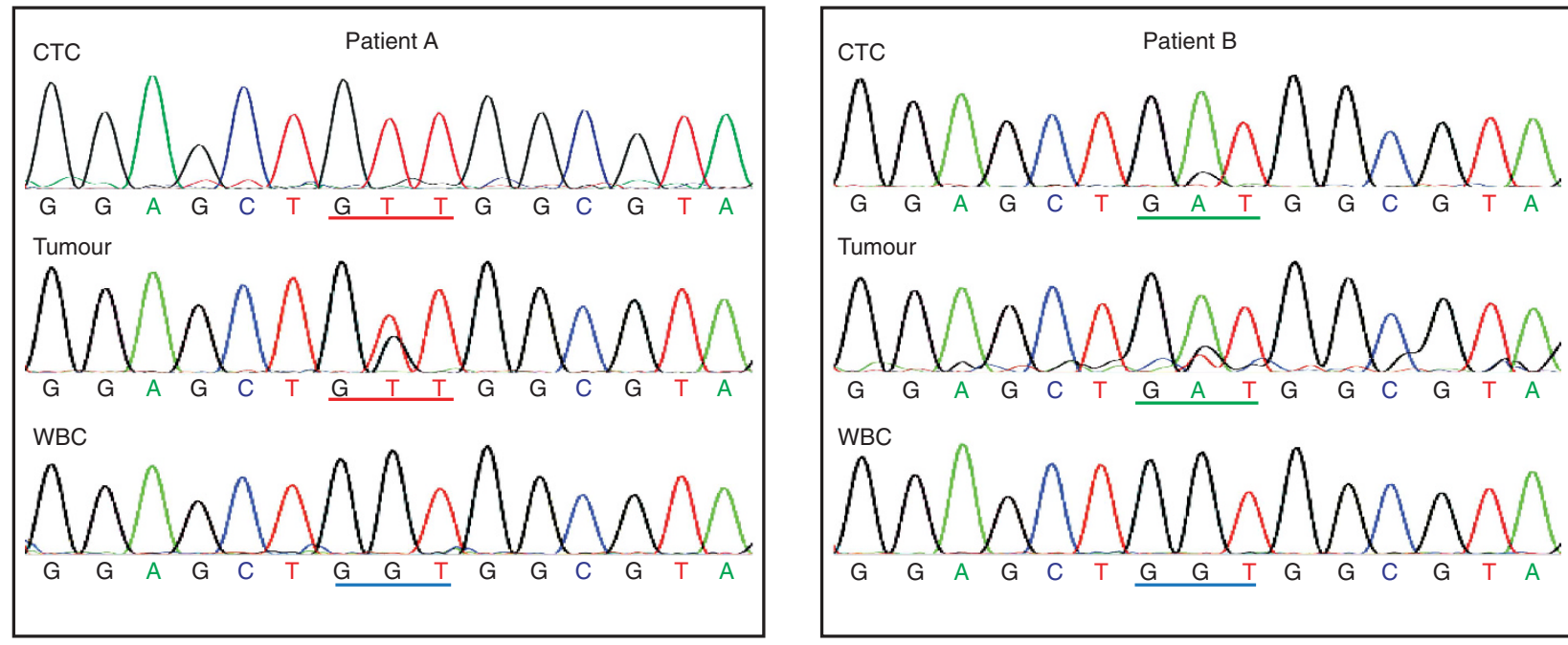

Tumour

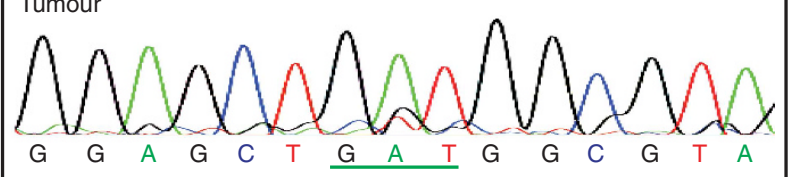
WBC

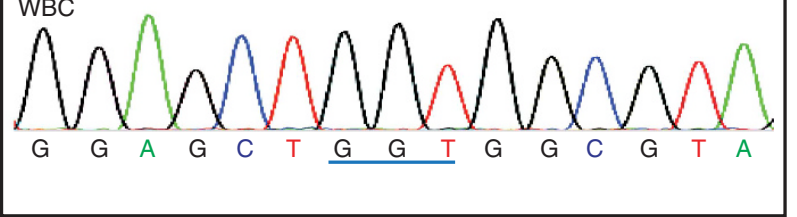

Codon 12 mutation subtype

G12V

G12D

Wild type

Figure 3. Validation of ICC and evaluation of tumour origin for CTCs by KRAS mutational analysis. (A) Flow diagram depicting the confirmation of tumour origin by isolating and sequencing CTCs and patient matched primary tumour tissue. (B) Sanger sequencing results for KRAS codon-12 mutations. CTCs, WBCs, and primary tumour tissue for two of the patients are depicted. Patient A has a G12V mutation and patient B has a G12D mutation. Both patients' WBCs were found to have wild-type KRAS sequences.

of CTCs to discriminate PDAC from non-adenocarcinoma (Figure 4B).

CTCs correlation with PDAC stage. CTCs were found in 0.0, 60.7, 78.6, and $96.3 \%$ of AJCC stages I, II, III, and IV patients, respectively (Table 1 ). For these respective stage groups, median CTC numbers were $0($ range $=0), 1($ range $=0-6), 1($ range $=0-9)$, and 5 (range $=0-48$ ) per $4 \mathrm{ml} \mathrm{VB} \mathrm{(Figure} 5 \mathrm{~A}$ ). In comparing CTC counts by stage group, a statistically significant difference between stage-IV PDAC and all other stages of PDAC $(P<0.0001)$ was found on the multiple comparisons test, but no differences were noted between AJCC stages I, II, or III groups. In the stage-II patients with available node status, there was no statistically significant difference in CTC count between node 

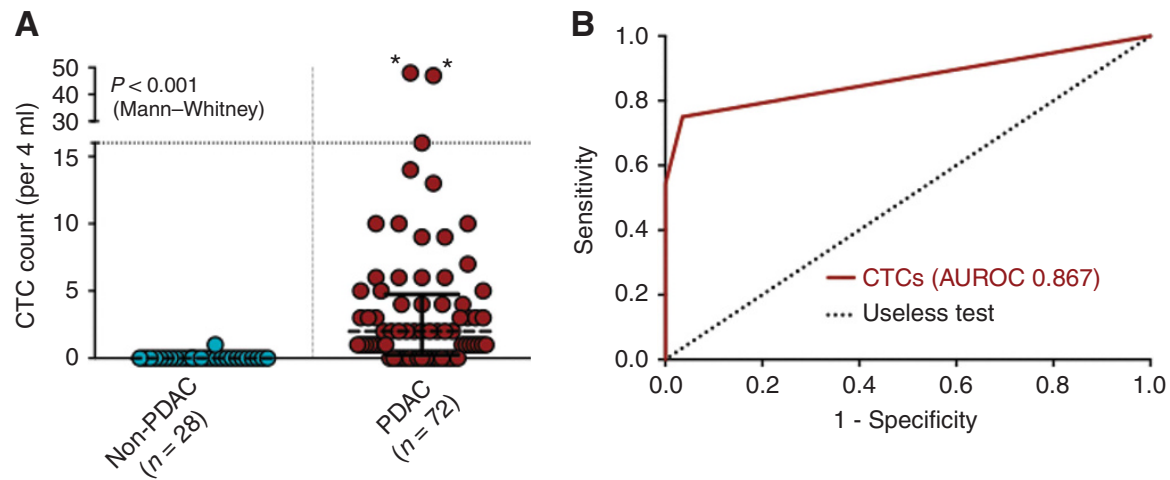

Figure 4. CTCs as a diagnostic biomarker. (A) Comparison of CTC enumeration in PDAC and non-adenocarcinoma diseases. (B) ROC curve for illustration of CTC performance in the discrimination of PDAC from non-adenocarcinoma diseases. CTC AUROC $=0.867(95 \% \mathrm{Cl} 0.798-0.935$, $P<0.001)$.
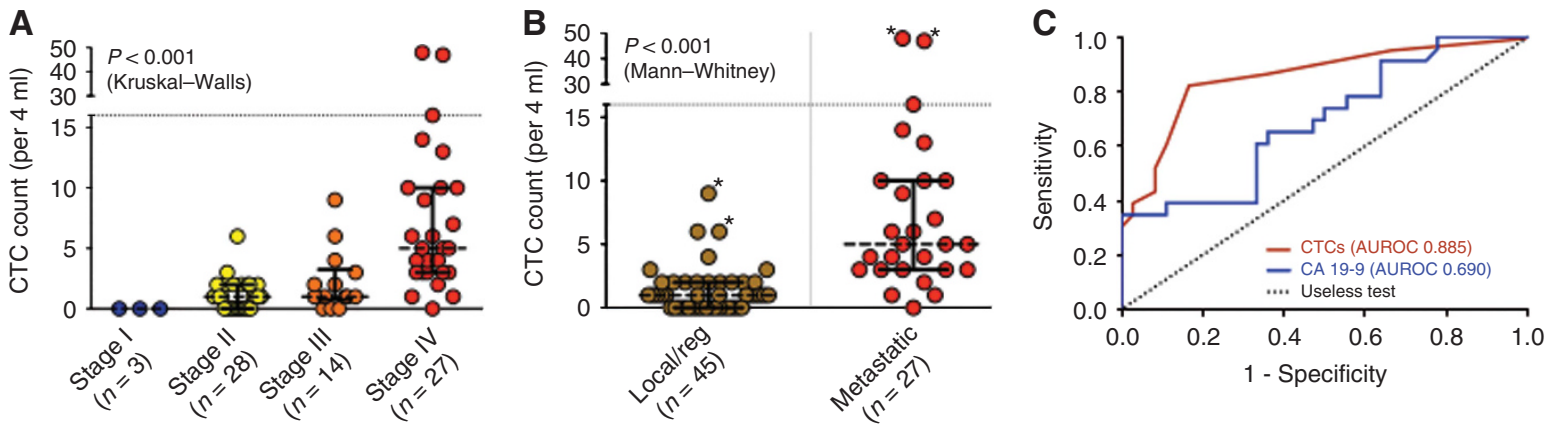

Figure 5. CTCs as a staging biomarker. (A) CTC enumeration showing correlation with PDAC stage. (B) CTC enumeration in local/regional (stages I-III) and metastatic (stage IV) disease. (C) Comparison of the performance of CTCs and CA19-9, in discriminating local/regional from metastatic disease. The CTC AUROC was $0.885(95 \% \mathrm{Cl}=0.800-0.969$ and $P<0.001)$, the CA19-9 AUROC was $0.690(95 \% \mathrm{Cl}=0.551-0.829$ and $P=0.014)$.

positive and node negative patients (Supplementary Figure S6A). In addition, CTC counts were not significantly different between patients with different tumour sizes or grades (Supplementary Figure S6B and C).

CTCs and pre-treatment staging. To test the ability of CTCs to differentiate clinically meaningful groups of patients, we compared those with local/regional (stages I-III) and metastatic (stage-IV) disease, and found a significant difference in CTC enumeration between these groups $(P<0.001$; Figure 5B). Based on J, a CTC value of $\geqslant 3$ CTCs in 4 -ml blood was the optimum cut-off for association with metastatic disease in patients with PDAC (Supplementary Table S4). At this cut-off value, CTCs showed a sensitivity of $85.2 \%$, specificity of $86.7 \%$, PPV of $79.3 \%$, and NPV of $90.7 \%$ in identifying patients with systemic disease and patients with $\geqslant 3$ CTCs per 4 -ml blood were 6.39 times more likely to harbour metastatic disease at the time of diagnosis. Overall, CTCs had an AUROC of 0.885 (95\% CI $=0.800-0.969$ and $P<0.001)$ for discriminating local/regional from metastatic disease (Figure $5 \mathrm{C}$ ). By comparison, in the 59/72 patients with available CA19-9 levels, use of the optimum cut-off of $\geqslant 3000$ units $\mathrm{ml}^{-1}$ yielded a sensitivity of $34.8 \%$, specificity of $100 \%$, PPV of $100 \%$, NPV of $70.6 \%$, and an AUROC of 0.690 (95\% CI $=0.551-0.829$ and $P=0.014)$ for the identification of PDAC patients with metastatic disease (Figure 5C).

Outlier analysis of CTCs for diagnosis and staging of PDAC. Finally, we performed an outlier analysis of our CTC enumeration in patients with PDAC to determine if our results were significantly impacted by extreme values. These values are marked with an asterisk in our CTC enumeration graphs (Figures 4 and 5).
For diagnosis of PDAC, exclusion of outliers did not significantly affect our data. The median CTC count of the PDAC group remained at 2, and the AUROC was still 0.863 (95\% CI $=0.793-$ 0.933 and $P<0.001)$. Similarly, the exclusion of outliers actually improved the AUROC for local/regional vs metastatic disease, new AUROC 0.902 (95\% CI $=0.818-0.986$ and $P<0.001)$. These results further support our finding of the ability of CTC presence and enumeration to differentiate patients with cancer from those without, and to identify which PDAC patients are more likely to harbour advanced AJCC stage disease.

\section{DISCUSSION}

Pancreatic cancer is a devastating disease that is incurable for $\sim 95 \%$ of those diagnosed (Siegel et al, 2015). Current diagnostic methods utilising image-guided biopsy are inconvenient, have associated morbidity, are relatively expensive and require local expertise. Furthermore, clinical staging based on cross-sectional imaging is insensitive to small-volume or micrometastatic disease and potentially results in under-staging at the time of diagnosis. This often results in these patients undergoing surgery as first-line therapy when, in fact, they have systemic disease. Surgery as firstline therapy carries significant morbidity and has been shown to cause delays in administration of systemic therapy, the most effective first-line therapy for metastatic disease (Merkow et al, 2014). Thus, there is a need for biomarkers that can help efficiently establish diagnosis and provide information regarding stage/ prognosis at the time of disease presentation to better inform initial therapeutic decisions. 
In the present study, we investigated CTCs as an adjunct biomarker for diagnosis and staging of PDAC at the time of disease presentation. We developed a sensitive and specific method for PDAC CTC detection using the NanoVelcro platform in conjunction with high-resolution fluorescent microscopy and a multi-colour ICC approach. We found the addition of CEA immunostaining to be of little value on its own, as only $1.6 \%$ of our CTCs isolated were CEA + /CK-/CD45-/Nucleus + . However, as an additional marker, we found it to be helpful for reaffirming the identity of CK + CTCs. We validated our CTC definition using NanoVelcro/LCM by mutational analysis of KRAS codon 12, demonstrating concordance of KRAS mutation subtype between CTCs and primary tumour tissue. We then demonstrated the ability of CTCs to function as an adjunctive biomarker for the diagnosis and staging of PDAC.

In regards to diagnosis of PDAC, our study found CTC presence $(\geqslant 1 \mathrm{CTC} / 4-\mathrm{ml} \mathrm{VB})$ performed with an overall sensitivity of $75.0 \%$ and specificity of $95.7 \%$. Studies by Kurihara et. al., Khoja et. al., and Bidard et. al., all utilised the CellSearch platform to study CTC enumeration in only advanced (stage III or IV) PDAC patients and found CTC presence in $42.3,39.6$, and $11 \%$ of patients, respectively (Kurihara et al, 2008; Khoja et al, 2012; Bidard et al, 2013). Our reported sensitivity was significantly higher than these studies, as we detected CTCs in 11/14 (78.6\%) stage III patients and 26/27 $(96.3 \%)$ stage IV patients. In fact, the sensitivity for our entire study cohort was higher, even with analysis of smaller blood volumes $(4 \mathrm{ml} v s 7.5 \mathrm{ml})$ and inclusion of early stage I/II patients ( $43.1 \%$ of our study cohort). This may be secondary to the higher sensitivity of microfluidic capture techniques for PDAC CTCs. For example, similar to our finding from stage IV patients, studies using the microfluidic CTC-Chip platform detected CTCs in 15/15 (100\%) stage IV PDAC patients (Nagrath et al, 2007; Yu et al, 2012). Another study utilising microfluidics detected high numbers of CTCs in both resectable and metastatic PDAC patients; however, they only reported the summary statistics of CTCs found, not the percentage of patients with CTCs. (Kamande et al, 2013) Using a different microfluidic device, Rhim et. al., found circulating pancreatic cells from 8/11 (73\%) PDAC patients with stages I-IV, similar to our $75 \%$ sensitivity (Rhim et al, 2014). Interestingly, they also found circulating pancreatic cells in $7 / 21$ (33\%) of patients with cystic pancreatic lesions, raising the possibility of EMT cells circulating in the blood as well (Rhim et al, 2012). Another promising technique is the isolation by size of epithelial tumour cell (ISET) method. A study by Khoja et al found CTCs in $\geqslant 90 \%$ of stage IV patients using ISET, similar to our sensitivity of $96.3 \%$ for stage IV patients (Khoja et al, 2012). All studies, including ours, had a reported specificity of $>90 \%$, similar to research of CTCs in other solid tumours that has consistently found CTCs to have high specificity (Court et al, 2015). Utilising mutational analysis as a means of confirming our CTC definition represents an orthogonal approach to ICC validation, and gave us confidence in our results. This is especially important for diagnostic tests, as a single CTC can only be considered diagnostic of a cancer if its tumour origin can be inferred.

The ability to accurately discriminate advanced disease is of obvious importance; informing first-line therapy, improving prognostication, and allowing trial stratification. In our study, CTC enumeration correlated with AJCC stage groups, and was further found to outperform CA19-9 as a biomarker for differentiating local/regional from metastatic disease. While further studies are needed, CTC enumeration may have potential as a prognostic biomarker signifying likely metastatic disease.

Our identification of KRAS mutations in CTCs, but not WBCs, makes us confident in our ability to distinguish CTCs from other circulating hematopoietic cells. Furthermore, we used KRAS mutational analysis of CTCs and matched tumour tissue to provide us with insight into the origin of the CTCs found.
Despite these confirmatory studies, we still had a single falsepositive result. This false-positive result occurred in a patient with a large $(6 \mathrm{~cm})$, complex cyst, non-diagnostic cyst fluid analysis, and lack of malignant cells on FNA. Although the patient may have a benign cyst, it is also possible that this patient may have malignant cystic disease. In addition to the risk from the cyst itself, studies have shown that $2.8-9.3 \%$ of patients with benign pancreatic cysts have synchronous or metachronous PDAC (Tanno et al, 2010; Lafemina et al, 2013). There is no available confirmatory pathology at the time of this publication as the patient chose not to pursue surgery. Of note, a recent study (Rhim et al, 2014) also demonstrated CTC presence in $\sim 30 \%$ of patients with premalignant pancreatic cystic disease utilising a different microfluidic technology and CTC identification criteria.

An important limitation of our study was the use of an epithelial surface marker (EpCAM) for CTC capture that potentially led to decreased sensitivity secondary to loss of CTCs expressing nonepithelial surface markers (Khoja et al, 2012). Given that PDAC cells have been shown to undergo epithelial to mesenchymal transition after entering the circulation in a mouse model, we hope to capture mesenchymal-type CTCs in the future as demonstrated in a study by Rhim et. al. (Rhim et al, 2012). These CTCs may provide different biological insights for pancreatic cancer. However, to our knowledge, our cohort is the largest to date looking at CTC enumeration as a biomarker for diagnosis and staging. This study is still on-going, and we hope to validate our findings in a larger cohort of patients. Furthermore, we have continued to follow all enrolled patients, and hope to analyse CTCs as a prognostic biomarker for recurrence and survival in future studies. In addition, our confirmation of tumour origin of captured CTCs demonstrates the potential for CTCs to function as a 'liquid biopsy' in PDAC. Future studies utilising technologies such as NanoVelcro/LCM should allow for comprehensive CTC molecular analysis, which may provide more biological insight into the intravasated tumour cell population as well as tumour heterogeneity and the mechanism of metastasis (Yu et al, 2012). Thus, future studies will hopefully not only confirm CTCs utility as a biomarker, but also demonstrate their potential to provide actionable information about the tumour's biology, which together holds great promise with respect to realising 'personalised' treatment of PDAC.

\section{ACKNOWLEDGEMENTS}

This work was funded in part by a UCLA Jonsson Comprehensive Cancer Impact Grant as well as an award provided by NantOmics. We would like to thank Dr William Isacoff for assistance in obtaining stage IV patient samples that allowed for the initial calibrations of the platform for pancreatic cancer CTC identification. The NanoVelcro Chips used in this research were supported by research grants (R33CA174562 and U01 CA198900) and an SBIR grant (R44 CA180482) from National Institute of Health.

\section{CONFLICT OF INTEREST}

Hsian-Rong Tseng, Co-Founder, Cytolumina Technologies Corp. Inc. The remaining authors declare no conflict of interest.

\section{REFERENCES}

Alix-Panabieres C, Pantel K (2014) Challenges in circulating tumour cell research. Nat Rev Cancer 14(9): 623-631.

Bidard FC, Huguet F, Louvet C, Mineur L, Bouche O, Chibaudel B, Artru P, Desseigne F, Bachet JB, Mathiot C, Pierga JY, Hammel P (2013) Circulating tumor cells in locally advanced pancreatic adenocarcinoma: 
the ancillary CirCe 07 study to the LAP 07 trial. Ann Oncol 24(8): 2057-2061.

Bryant KL, Mancias JD, Kimmelman AC, Der CJ (2014) KRAS: feeding pancreatic cancer proliferation. Trends Biochem Sci 39(2): 91-100.

Cameron JL, He J (2015) Two thousand consecutive pancreaticoduodenectomies. J Am Coll Surg 220(4): 530-536.

Chen JF, Ho H, Lichterman J, Lu YT, Zhang Y, Garcia MA, Chen SF, Liang AJ, Hodara E, Zhau HE, Hou S, Ahmed RS, Luthringer DJ, Huang J, Li KC, Chung LW, Ke Z, Tseng HR, Posadas EM (2015) Subclassification of prostate cancer circulating tumor cells by nuclear size reveals very small nuclear circulating tumor cells in patients with visceral metastases. Cancer 121(18): 3240-3251.

Cooper M, Newman NA, Ibrahim AM, Lam E, Herman JM, Singh VK, Wolfgang CL, Pawlik TM, Cameron JL, Makary MA (2013) Unnecessary tests and procedures in patients presenting with solid tumors of the pancreas. J Gastrointest Surg 17(7): 1218-1223.

Court CM, Ankeny JS, Hou S, Tseng HR, Tomlinson JS (2015) Improving pancreatic cancer diagnosis using circulating tumor cells: prospects for staging and single-cell analysis. Expert Rev Mol Diagn 15(11): 1491-1504.

Cristofanilli M, Budd GT, Ellis MJ, Stopeck A, Matera J, Miller MC, Reuben JM, Doyle GV, Allard WJ, Terstappen LW, Hayes DF (2004) Circulating tumor cells, disease progression, and survival in metastatic breast cancer. $N$ Engl J Med 351(8): 781-791.

Diehl SJ, Lehmann KJ, Sadick M, Lachmann R, Georgi M (1998) Pancreatic cancer: value of dual-phase helical CT in assessing resectability. Radiology 206(2): 373-378.

Girgis MD, Olafsen T, Kenanova V, McCabe KE, Wu AM, Tomlinson JS (2011) Targeting CEA in pancreas cancer xenografts with a mutated scFv-Fc antibody fragment. EJNMMI Res 1(1): 24.

Hawes RH, Xiong Q, Waxman I, Chang KJ, Evans DB, Abbruzzese JL (2000) A multispecialty approach to the diagnosis and management of pancreatic cancer. Am J Gastroenterol 95(1): 17-31.

Hou S, Zhao L, Shen Q, Yu J, Ng C, Kong X, Wu D, Song M, Shi X, Xu X, OuYang WH, He R, Zhao XZ, Lee T, Brunicardi FC, Garcia MA, Ribas A, Lo RS, Tseng HR (2013) Polymer nanofiberembedded microchips for detection, isolation, and molecular analysis of single circulating melanoma cells. Angew Chem Int Ed Engl 52(12): 3379-3383.

Kamande JW, Hupert ML, Witek MA, Wang H, Torphy RJ, Dharmasiri U, Njoroge SK, Jackson JM, Aufforth RD, Snavely A, Yeh JJ, Soper SA (2013) Modular microsystem for the isolation, enumeration, and phenotyping of circulating tumor cells in patients with pancreatic cancer. Anal Chem 85(19): 9092-9100.

Khoja L, Backen A, Sloane R, Menasce L, Ryder D, Krebs M, Board R, Clack G, Hughes A, Blackhall F, Valle JW, Dive C (2012) A pilot study to explore circulating tumour cells in pancreatic cancer as a novel biomarker. Br J Cancer 106(3): 508-516.

Kurihara T, Itoi T, Sofuni A, Itokawa F, Tsuchiya T, Tsuji S, Ishii K, Ikeuchi N, Tsuchida A, Kasuya K, Kawai T, Sakai Y, Moriyasu F (2008) Detection of circulating tumor cells in patients with pancreatic cancer: a preliminary result 15. J Hepatobiliary Pancreat Surg 15(2): 189-195.

Lafemina J, Katabi N, Klimstra D, Correa-Gallego C, Gaujoux S, Kingham TP, Dematteo RP, Fong Y, D’Angelica MI, Jarnagin WR, Do RK, Brennan MF, Allen PJ (2013) Malignant progression in IPMN: a cohort analysis of patients initially selected for resection or observation. Ann Surg Oncol 20(2): 440-447.
LeBlanc JK, Ciaccia D, Al-Assi MT, McGrath K, Imperiale T, Tao LC, Vallery S, DeWitt J, Sherman S, Collins E (2004) Optimal number of EUS-guided fine needle passes needed to obtain a correct diagnosis. Gastrointest Endosc 59(4): 475-481.

Merkow RP, Bilimoria KY, Tomlinson JS, Paruch JL, Fleming JB, Talamonti MS, Ko CY, Bentrem DJ (2014) Postoperative complications reduce adjuvant chemotherapy use in resectable pancreatic cancer. Ann Surg 260(2): 372-377.

Nagrath S, Sequist LV, Maheswaran S, Bell DW, Irimia D, Ulkus L, Smith MR, Kwak EL, Digumarthy S, Muzikansky A, Ryan P, Balis UJ, Tompkins RG, Haber DA, Toner M (2007) Isolation of rare circulating tumour cells in cancer patients by microchip technology. Nature 450(7173): 1235-1239.

Rahib L, Smith BD, Aizenberg R, Rosenzweig AB, Fleshman JM, Matrisian LM (2014) Projecting cancer incidence and deaths to 2030: the unexpected burden of thyroid, liver, and pancreas cancers in the United States. Cancer Res 74(11): 2913-2921.

Rhim AD, Mirek ET, Aiello NM, Maitra A, Bailey JM, McAllister F, Reichert M, Beatty GL, Rustgi AK, Vonderheide RH, Leach SD, Stanger BZ (2012) EMT and dissemination precede pancreatic tumor formation. Cell 148(1-2): 349-361.

Rhim AD, Thege FI, Santana SM, Lannin TB, Saha TN, Tsai S, Maggs LR, Kochman ML, Ginsberg GG, Lieb JG, Chandrasekhara V, Drebin JA, Ahmad N, Yang YX, Kirby BJ, Stanger BZ (2014) Detection of circulating pancreas epithelial cells in patients with pancreatic cystic lesions. Gastroenterology 146(3): 647-651.

Siegel RL, Miller KD, Jemal A (2015) Cancer statistics, 2015. CA Cancer J Clin 65(1): 5-29.

Tanno S, Nakano Y, Sugiyama Y, Nakamura K, Sasajima J, Koizumi K, Yamazaki M, Nishikawa T, Mizukami Y, Yanagawa N, Fujii T, Obara T, Okumura T, Kohgo Y (2010) Incidence of synchronous and metachronous pancreatic carcinoma in 168 patients with branch duct intraductal papillary mucinous neoplasm. Pancreatology 10(2-3): 173-178.

Wang S, Liu K, Liu J, Yu ZT, Xu X, Zhao L, Lee T, Lee EK, Reiss J, Lee YK, Chung LW, Huang J, Rettig M, Seligson D, Duraiswamy KN, Shen CK, Tseng HR (2011) Highly efficient capture of circulating tumor cells by using nanostructured silicon substrates with integrated chaotic micromixers. Angew Chem Int Ed Engl 50(13): 3084-3088.

Yachida S, Jones S, Bozic I, Antal T, Leary R, Fu B, Kamiyama M, Hruban RH, Eshleman JR, Nowak MA, Velculescu VE, Kinzler KW, Vogelstein B, Iacobuzio-Donahue CA (2010) Distant metastasis occurs late during the genetic evolution of pancreatic cancer. Nature 467(7319): 1114-1117.

Yu M, Ting DT, Stott SL, Wittner BS, Ozsolak F, Paul S, Ciciliano JC, Smas ME, Winokur D, Gilman AJ, Ulman MJ, Xega K, Contino G, Alagesan B, Brannigan BW, Milos PM, Ryan DP, Sequist LV, Bardeesy N, Ramaswamy S, Toner M, Maheswaran S, Haber DA (2012) RNA sequencing of pancreatic circulating tumour cells implicates WNT signalling in metastasis. Nature 487(7408): 510-513.

This work is published under the standard license to publish agreement. After 12 months the work will become freely available and the license terms will switch to a Creative Commons AttributionNonCommercial-Share Alike 4.0 Unported License.

Supplementary Information accompanies this paper on British Journal of Cancer website (http://www.nature.com/bjc) 